\title{
Acquired resistance to combination treatment through loss of synergy with MEK and PI3K inhibitors in colorectal cancer
}

\author{
Bhaskar Bhattacharya ${ }^{1}$, Sarah Hong Hui Low ${ }^{1}$, Mei Ling Chong ${ }^{1}$, Dilys Chia ${ }^{2}$, King \\ Xin Koh ${ }^{3}$, Nur Sabrina Sapari ${ }^{1}$, Stanley Kaye ${ }^{4}$, Huynh Hung ${ }^{5}$, Touati Benoukraf ${ }^{1}$, \\ Richie Soong ${ }^{1,3}$ \\ ${ }^{1}$ Cancer Science Institute of Singapore, National University of Singapore, Singapore \\ ${ }^{2}$ Department of Pharmacy, National University of Singapore, Singapore \\ ${ }^{3}$ Department of Pathology, National University of Singapore, Singapore \\ ${ }^{4}$ Drug Development Unit, Royal Marsden NHS Trust, The Royal Marsden NHS Foundation Trust, London, United Kingdom \\ ${ }^{5}$ Laboratory of Molecular Endocrinology, National Cancer Centre of Singapore, Singapore
}

Correspondence to: Richie Soong, e-mail: richie.soong@nus.edu.sg Bhaskar Bhattacharya, e-mail: csibbu@nus.edu.sg

Keywords: acquired resistance, combination therapy, MEK inhibitors, PI3K inhibitors, synergy

Received: December 01, 2015

Accepted: March 28, 2016

Published: April 11, 2016

\section{ABSTRACT}

Historically, understanding of acquired resistance (AQR) to combination treatment has been based on knowledge of resistance to its component agents. To test whether an altered drug interaction could be an additional factor in AQR to combination treatment, models of AQR to combination and single agent MEK and PI3K inhibitor treatment were generated. Combination indices indicated combination treatment of PI3K and MEK inhibitors remained synergistic in cells with AQR to single agent but not combination AQR cells. Differences were also observed between the models in cellular phenotypes, pathway signaling and drug cross-resistance. Genomics implicated TGFB2-EDN1 overexpression as candidate determinants in models of $A Q R$ to combination treatment. Supplementation of endothelin in parental cells converted synergism to antagonism. Silencing of TGFB2 or EDN1 in cells with AQR conferred synergy between PI3K and MEK inhibitor. These results highlight that AQR to combination treatment may develop through alternative mechanisms to those of single agent treatment, including a change in drug interaction.

\section{INTRODUCTION}

Combination therapy, entailing treatment using two or more agents together, has become a common strategy for optimizing treatment efficacy, and reducing toxicity and drug resistance [1]. The strategy is based on the principle that an additional effect is generated by combining agents over using them individually. These effects can be synergistic, additive, or antagonistic, namely greater than, equal to, or worse than the added effects of the component agents respectively.

Drug resistance remains a major cause of treatment failure [2]. The resistance can be due to intrinsic factors that manifest in a lack of initial drug activity. Resistance can also be acquired, developing as a result of changes induced by the selection pressure of drug treatment. Many mechanisms of acquired resistance (AQR) have been identified, including changes in drug efflux, metabolism and detoxification, and acquired DNA mutations, gene amplification, pathway redundancy, crosstalk and feedback, and the enrichment of resistant subclones [3].

In recent times, the emergence of moleculartargeted therapy has led to an increased consideration of combination therapy as a means of circumventing drug resistance. However, AQR to combination therapy can still be expected, with evidence readily available from the clinic. What is unclear is whether mechanisms of $\mathrm{AQR}$ to combination therapy are the same as those for monotherapy, and whether an altered drug interaction could also be a factor. In this study, models of AQR to the prominent combination of MEK and PI3K inhibitors [4-9] were generated concurrently with models to single agent treatment. Selective loss of synergy in combination treatment was observed in the cells with AQR to 
combination treatment but not single agent treatment, presenting loss of synergy as a novel mechanism of AQR to be considered in the application of combination therapy.

\section{RESULTS}

\section{Generation of AQR and loss of synergy}

HCT116 cells with KRAS G13D and PIK3CA H1047R mutations (cancer.sanger.ac.uk) were cultured in the presence of both AZD6244 (MEK inhibitor) and BKM120 (PI3K inhibitor) at $\mathrm{IC}_{50}$ concentrations of each agent, AZD6244 alone (2 treatments of $1 / 2$ IC50 concentrations), BKM120 alone (2 treatments of $1 / 2$ $\mathrm{IC}_{50}$ concentrations), or vehicle ( 2 treatments of $0.25 \%$ DMSO). Two treatments were provided for all models to minimize bias from the number of treatments of the cells.

After prolonged treatment, HCT116 cells cultured with both AZD6244 and BKM120 became resistant to combination AZD6244 and BKM120 treatment (designated as "HCT116CR" cells) compared to HCT116 cells cultured with DMSO ("HCT116DM" cells) (Table 1). Combination index (CI) analysis [10] indicated that AZD6244 and BKM120 were antagonistic in HCT116CR cells, while they were synergistic in HCT116DM cells. HCT116CR cells also displayed increased resistance to single agent treatment with AZD6244, but not BKM120.

HCT116 cells treated with AZD6244 alone ("HCT116AR" cells) and BKM120 alone ("HCT116BR" cells) displayed AQR to their respective treatments. Crossresistance was observed for HCT116AR cells to BKM120, as well as for HCT116BR cells to AZD6244. Nonetheless, the combination of AZD6244 and BKM120 remained synergistic in HCT116AR and HCT116BR cells.

To confirm that the AQR and loss of synergy was not compound specific, the sensitivity of the cells to GDC0973 (MEK inhibitor) and BYL719 (PI3K inhibitor) treatment was assessed. Similar patterns of AQR, crossresistance and loss of synergy was observed with these agents in respective cells (Table 1). The only difference in pattern was an increased resistance of HCT116CR cells to BYL719.

To confirm that the observations were not specific to HCT116 cells, LoVo (KRAS G13D mutant, cancer. sanger.ac.uk) colorectal cancer cells with AQR to AZD6244 ("LoVoAR"), BKM120 ("LoVoBR") and their combination ("LoVoCR") were generated using the same methods applied to HCT116 cells. The cells exhibited similar patterns of resistance to AZD6244 and BKM120 treatment, as well as GCD0973 and BYL719 treatment, as observed for HCT116 cells (Supplementary Table S1).

\section{Pathway signaling and inhibition}

Analysis of baseline p-Erk, p-Akt, p-S6 and p-4EBP1 revealed HCT116AR cells had higher levels of p-Erk than HCT116DM cells (Figure 1), consistent with a previous report [11]. HCT116BR cells had elevated p-Erk and p-Akt. HCT116CR cells also had increased p-Erk and p-Akt, but also reduced p-4EBP1.

Following combination treatment, p-Erk, p-Akt, p-S6 and p-4EBP1 were reduced in all cells, indicating pathway inhibition activity was retained. AZD6244 treatment also reduced p-Erk in all cells, and BKM120 treatment reduced p-Akt in all cells, indicating that the inhibitory activity of single agents was retained as well. BKM120 also reduced p-4EBP1 in HCT116CR and HCT116AR but not HCT116BR cells, suggesting the AQR of HCT116BR cells to PI3K inhibition could involve reduced p-4EBP1 inhibition. AZD6244 also significantly reduced p-Akt, and p-4EBP1 (not statistically significant; $p=0.06)$ in HCT116CR but not the other cells.

\section{Cell phenotype analysis}

Consistent with their known activity $[12,13]$, single agent AZD6244 and BKM120 treatment led to increased G1 phase populations, and combination treatment led to an increased sub-G1 population and apoptosis in HCT116DM cells (Figure 2). In HCT116AR and HCT116BR cells, the increase in G1 phase populations was not observed following single agent treatment. However, combination treatment still led to an increased sub-G1 population and apoptosis in these cells. In HCT116CR cells, there was no significant increase in G1 or sub-G1 populations or apoptosis following single agent and combination treatment (Supplementary Figure S1). Indeed, apoptosis after combination treatment was significantly reduced in HCT116CR cells compared to HCT116DM cells. In wound healing experiments, HCT116AR, HCT116BR and HCT116CR cells displayed increased migration compared to HCT116DM cells when treated with DMSO. Combination treatment led to a reduction in migration of HCT116DM, HCT116AR and HCT116BR, but not HCT116CR cells.

\section{Drug cross-resistance analysis}

No significant differences were observed in the sensitivity of HCT116-derived cells to 5-Fluorouracil, carboplatin and sorafenib, suggesting that a multi-drug resistance phenotype was not responsible for resistance (Supplementary Table S2). Consistent with the earlier observations, HCT116AR, HCT116BR and HCT116CR cells were more resistant than HCT116DM cells to an extended panel of MEK and PI3K inhibitors. Interestingly, HCT116CR cells also exhibited sensitivity to Akt and 
Table 1: $\mathrm{IC}_{50}$ and combination index values of treatment with various drugs and their combinations in HCT116-derived cells

\begin{tabular}{|c|c|c|c|c|}
\hline Cell Line & HCT116DM & HCT116AR & HCT116BR & HCT116CR \\
\hline AZD6244 $\mathrm{IC}_{50}(\mu \mathrm{M})$ & $2.8 \pm 0.03$ & $30.2 \pm 0.12 *$ & $10.2 \pm 0.20^{*}$ & $6.1 \pm 0.31^{*}$ \\
\hline $\mathrm{BKM} 120 \mathrm{IC}_{50}(\mu \mathrm{M})$ & $1.3 \pm 0.01$ & $4.8 \pm 0.04 *$ & $5.2 \pm 0.19^{*}$ & $1.2 \pm 0.02$ \\
\hline AZD6244 + BKM120 IC $50(\mu \mathrm{M})$ & $0.8 \pm 0.02$ & $0.9 \pm 0.04$ & $0.9 \pm 0.02$ & $10 \pm 2.34^{*}$ \\
\hline $\mathrm{AZD} 6244+\mathrm{BKM} 120 \mathrm{CI}_{\mathrm{fu} 0.5}$ & $0.15 \pm 0.031$ & $0.29 \pm 0.028$ & $0.21 \pm 0.007$ & $1.98 \pm 0.210^{*}$ \\
\hline $\mathrm{GDC} 0973 \mathrm{IC}_{50}(\mu \mathrm{M})$ & $5.7 \pm 0.81$ & $20.3 \pm 2.32 *$ & $22.1 \pm 1.94^{*}$ & $17.8 \pm 1.66^{*}$ \\
\hline $\mathrm{BYL} 719 \mathrm{IC}_{50}(\mu \mathrm{M})$ & $9.8 \pm 1.20$ & $30.2 \pm 3.11 *$ & $38.2 \pm 4.04^{*}$ & $25.6 \pm 2.85^{*}$ \\
\hline $\mathrm{GDC} 0973+\mathrm{BYL} 19 \mathrm{IC}_{50}(\mu \mathrm{M})$ & $0.5 \pm 0.05$ & $0.5 \pm 0.02$ & $0.6 \pm 0.05$ & $10.9 \pm 1.81 *$ \\
\hline GDC0973 + BYL719 CI $\mathrm{fu}_{\mathrm{fu} .5}$ & $0.16 \pm 0.083$ & $0.28 \pm 0.042$ & $0.39 \pm 0.012$ & $1.96 \pm 0.381^{*}$ \\
\hline
\end{tabular}

The $\mathrm{IC}_{50}$ values of AZD6244, BKM120, GDC0973, and BYL719 as single agents and in combination (in the presence of the other drug at fixed ratio of their $\mathrm{IC}_{50}$ values) are indicated. CI values for fraction unaffected at $\mathrm{IC}_{50}(\mathrm{fu} 0.5)$ are also given. Additivity $=1$, Antagonism $>1$, Synergy $<1$.

* $p<0.05$ for differences in $\mathrm{IC}_{50}$ values compared to HCT116DM, and for differences to 1 for CI values.

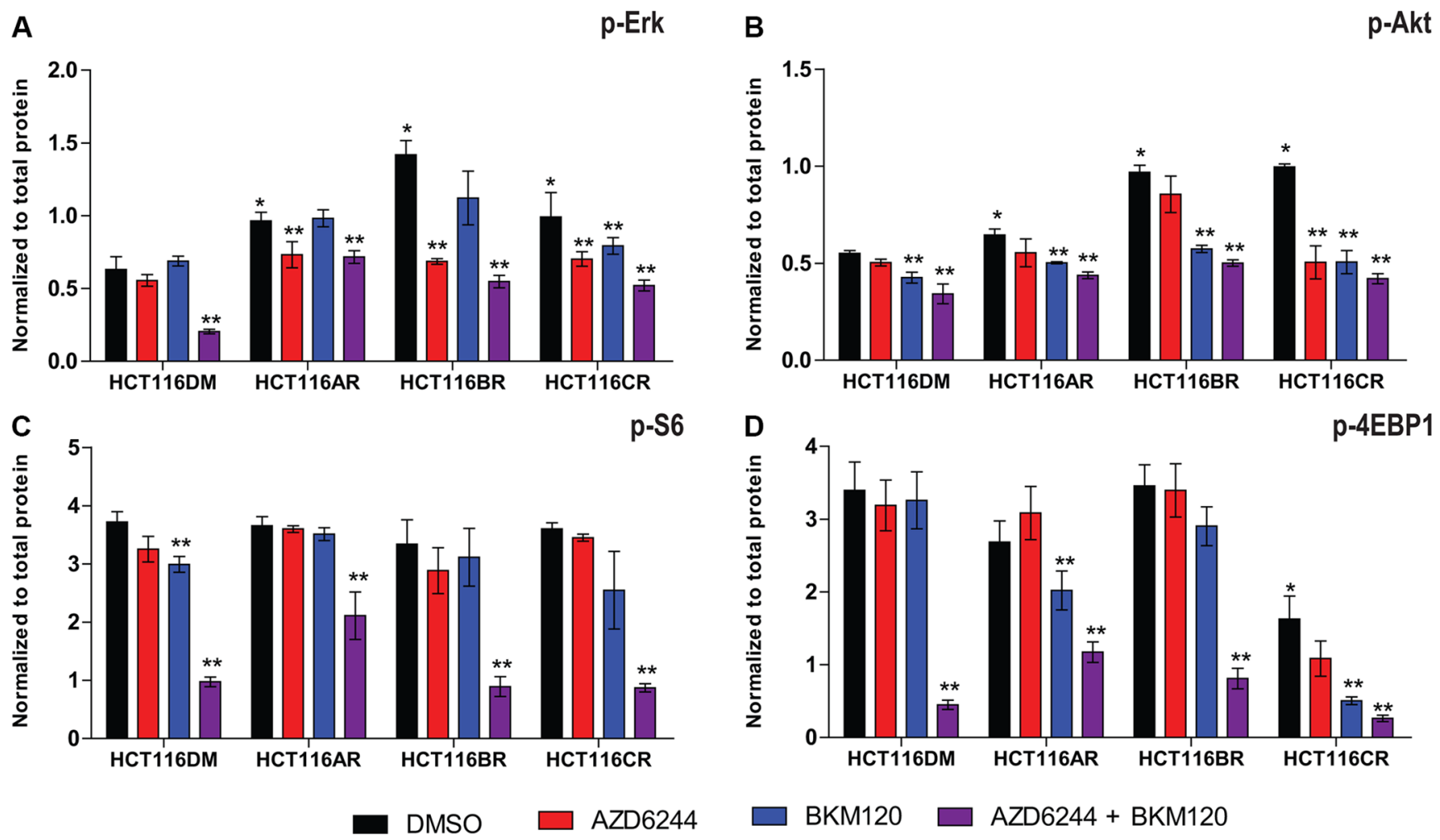

Figure 1: Pathway signaling levels of AQR cell lines. Phosphorylation levels of (A) Erk, (B) Akt, (C) S6 and (D) 4EBP1 at $24 \mathrm{~h}$ post-treatment in HCT116DM, HCT116AR, HCT116BR and HCT116CR cells treated with vehicle (DMSO), AZD6244 alone ( $\mathrm{IC}_{50}$ concentration), BKM120 alone ( $\mathrm{IC}_{50}$ concentration), and their combination $\left(\mathrm{IC}_{50}+\mathrm{IC}_{50}\right.$ concentration). Levels were measured by ELISA. All experiments were repeated three times, and data are displayed as mean \pm standard deviation of phosphorylated protein normalized to total protein. *indicates $p<0.05$ compared to levels in HCT116DM. ${ }^{*}$ *indicates $p<0.05$ compared to the control levels in the treated cell lines. 


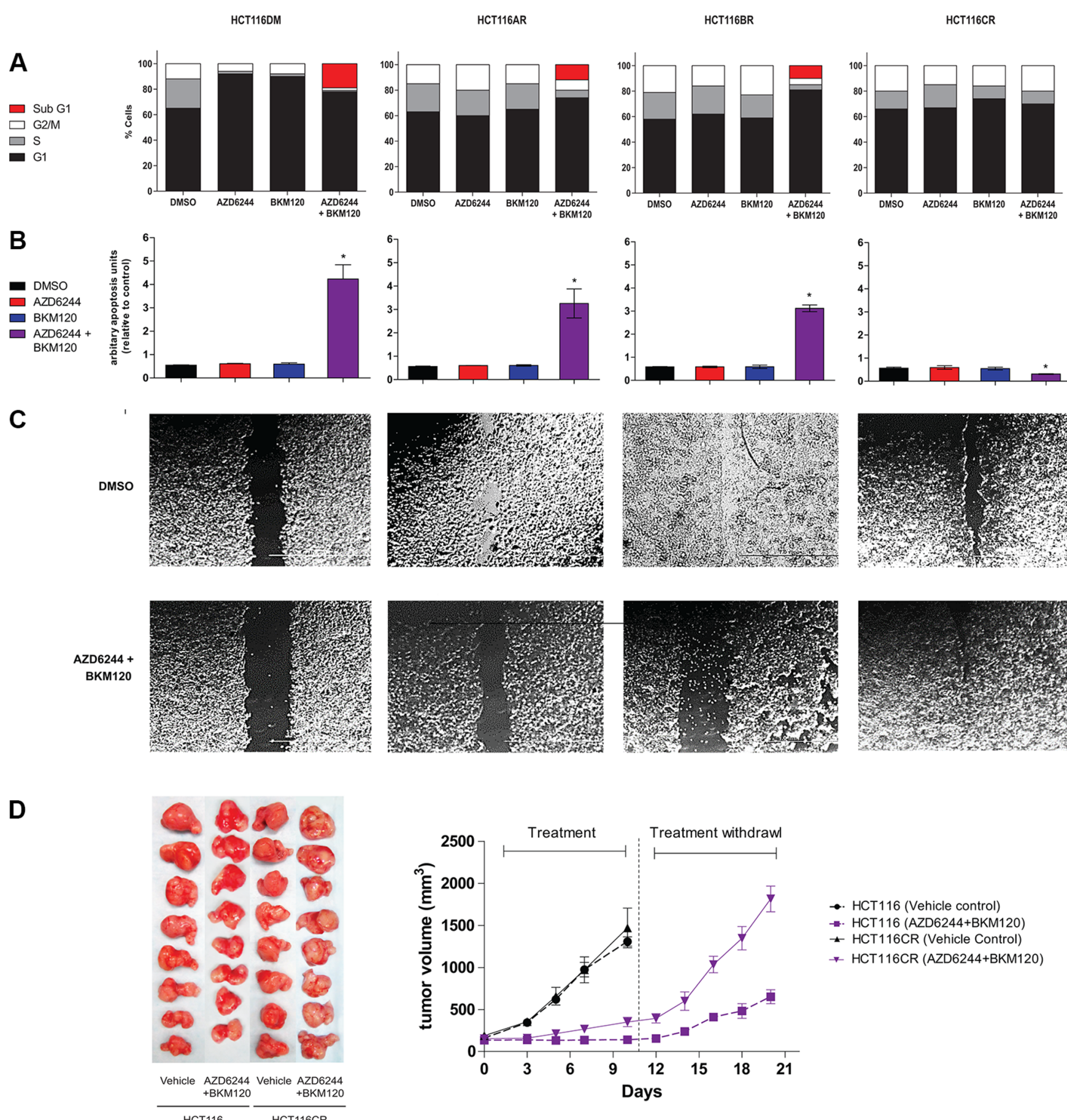

Figure 2: In-vitro and in-vivo phenotypes of AQR cell lines. (A) Cell cycle phase distribution of cells at baseline and $24 \mathrm{~h}$ after exposure to $\mathrm{IC}_{50}$ concentrations of AZD6244 alone, BKM120 alone and AZD6244 and BKM120 combined. (B) Apoptosis of cells at baseline and $24 \mathrm{~h}$ after exposure to $\mathrm{IC}_{50}$ concentrations of AZD6244 alone, BKM120 alone and AZD6244 and BKM120 combined. *indicates $p<0.05$ compared to cells treated with vehicle (DMSO). (C) Phase contrast images of cells in the wound-healing assay at baseline and $24 \mathrm{~h}$ after exposure to $\mathrm{IC}_{50}$ concentrations of AZD6244 alone, BKM120 alone and AZD6244 and BKM120 combined. (D) Images of tumors (left panel) and a chart of tumor volume over time (right panel) of tumors established from HCT116DM or HCT116CR cells in male SCID mice as described in online methods. All experiments were repeated three times, and data are displayed as mean \pm standard deviation where relevant. 
mTOR inhibitors. HCT116BR cells shared sensitivity with HCT116CR cells to Akt inhibitors. HCT116AR cells were more resistant to mTOR inhibitors.

\section{In vivo assessment}

HCT116CR and HCT116DM cells were implanted in male SCID mice as described in Methods. Treatments started when the tumors reached the size of approximately $130-150 \mathrm{~mm}^{3}$, and consisted of either treatment with vehicle or the combination of AZD6244 (25 mg/ $\mathrm{kg})$ and BKM120 (40 mg/kg) daily. Treatment ceased after 10 days when the tumors in the mice treated with vehicle reached their maximum allowed tumor volume. Consistent with the treatment synergy observed in-vitro, HCT116DM tumor growth was significantly inhibited by treatment with the combination compared to vehicle ( $p=0.01$, Figure 2 ). Consistent with the AQR observed in-vitro, combination treatment reduced tumor growth less in HCT116CR than HCT116DM tumors, however the difference was not statistically significant. After treatment withdrawal, tumors from mice receiving combination treatment were measured for an additional 10 days. Tumors from mice implanted with HCT116CR cells grew at a faster rate than those implanted with HCT116DM cells $(p=0.03)$.

\section{DNA variant analysis}

All 8 cells were screened for DNA variants in 50 prominent genes in cancer using the Ion AmpliSeq Cancer Hotspot Panel v2. The average depth of sequencing was $3,792 \pm 424$ reads and uniformity of base coverage was $98.53 \pm 0.01 \%$ (Supplementary Table S3). All variants in HCT116 and LoVo cells that were expected to be detected according to previous reports [14] and assay design were detected. Four additional variants were detected: SMAD4 (Y412H) in HCT116AR, TP53 (T18A) and TP53 (Y236C) in HCT116CR, and PTEN (A126S) in LoVoDM cells.

\section{RNA expression analysis}

Using gene expression arrays, eleven probes were differentially expressed (adjusted $p<0.01$ and foldchange $>1.5$ ) and consistent between HCT116CR and LoVoCR cells compared to the others (Supplementary Figure S2). Endothelin-1 (EDN1) and transforming growth factor beta 2 (TGFB2) had the highest differential expression (3.84 and 3.94 higher in CR cells respectively), of which the $p$-value was lowest for EDN1 $\left(1.22 \times 10^{-9}\right.$ and $2.44 \times 10^{-6}$ respectively). The significant difference in EDN1 and TGFB2 expression between CR and DM cells was verified by real-time PCR (Figure $3 \mathrm{~B}$ and $3 \mathrm{C}$ ) and western immunoblotting in both HCT116- and LoVoderived cells (Supplementary Figure S3). The increased expression of both TGFB2 and EDN1 was of particular interest given that TGFB2 can upregulate EDN1 through SMAD activation $[15,16]$. No changes in the expression of EDN1 and TGFB2 were observed in the single agent AQR cells, and expression of endothelin receptors also remained unchanged in both the combination AQR cells compared to parental cells (Supplementary Figure S4).

\section{Modulation of EDN1 and TGFB2}

To investigate the involvement of EDN1 in drug resistance, parental HCT116 were cultured with $100 \mathrm{nM}$ endothelin-1 or with vehicle for 24 hours before treatment with the combination of AZD6244 and BKM120. The two drugs were antagonistic in cells cultured with endothelin-1 $\left(\mathrm{CI}_{\text {fu0.5 }}=1.9 \pm 0.05\right)$ while they were synergistic in HCT 116 cells cultured with vehicle $(0.19 \pm 0.02)$. Cells cultured with endothelin-1 also had higher p-Akt, p-Erk and p-4EBP1 levels than those cultured with vehicle (Figure 3).

HCT116 and HCT116CR cells were also transfected with EDN1 and TGFB2 siRNA, and confirmed to have reduced $E D N 1$ and $T G F B 2$ RNA levels respectively compared to cells transfected with scramble siRNA. TGFB2 siRNA transfection also reduced EDN1 RNA levels, while EDN1 siRNA transfection did not reduce TGFB2 RNA levels, consistent with TGFB2 being a upstream regulator of EDN1 [15, 16]. Transfection of EDN1 siRNA in HCT116CR cells led to synergism between AZD6244 and BKM120 (0.52 \pm 0.03$)$, while antagonism remained in cells transfected with scrambled siRNA $(2.1 \pm 0.05)$. In parental HCT116 cells, the two drugs remained synergistic whether transfected with EDN1 siRNA $(0.25 \pm 0.04)$ or scrambled siRNA $(0.22 \pm 0.02)$. Similarly, transfection with TGFB2 siRNA $(0.62 \pm 0.08)$ but not scrambled siRNA $(1.9 \pm 0.07)$ also converted antagonism to synergism in HCT116CR cells. There was also no change in the synergism of combination treatment from the transfection of TGFB2 siRNA $(0.49 \pm 0.06)$ or scramble siRNA $(0.32 \pm 0.05)$ in HCT116 cells. EDN1 and TGFB2 siRNA also both reduced the levels of p-Akt and p-Erk in HCT116CR cells, but not HCT116 cells. The same results were observed from endothelin-1 and siRNA priming of LoVo-derived cells (Supplementary Figure S5). Additionally, synergy between PI3K and MEK inhibitors was also restored in HCT116CR $(\mathrm{CI} @$ fu0 $0.5=0.53 \pm 0.02$, $p<0.05)$ and LoVoCR $(\mathrm{CI} @ \mathrm{fu} 0.5=0.61 \pm 0.04, p<0.05)$ cells in the presence of a fixed-low growth inhibitory concentration ( $5 \%$ growth inhibition) of bosentan, a dual endothelin receptor antagonist (Supplementary Figure S6).

\section{DISCUSSION}

During the execution of this study, two controlled studies of AQR to combination treatment were reported. Ahronian et al. described AQR to combination BRAF and MEK inhibitor treatment in melanoma cells in-vitro that 
was associated with acquisition of $K R A S$ amplification [17]. KRAS amplification was also observed in a tumor at recurrence in a melanoma patient treated with combination BRAF and MEK inhibitor therapy. Pirazolli et al. reported on AQR to combination afatinib and cetuximab treatment in EGFR-mutant lung cancer in-vitro and in-vivo that was linked to acquisition of mTOR activation [18]. Similar resistance and aberration was observed in a lung cancer patient treated with afatinib and cetuximab.

A major element in the consideration of combination therapy however, is the effect of the drug interaction, be it synergistic, additive or antagonistic [19]. Traditionally, this interaction has been measured by the protocol of Chou and Talalay [19]. The protocol allows calculation of a $\mathrm{CI}$ value derived from $\mathrm{IC}_{50}$ values of respective drugs at fixed ratios over a concentration range. Through this approach, the nature of interaction of combined drugs can be quantified, and misinterpretation of "one-sided" enhancement as synergy can be avoided.

The primary hypothesis of this study was that an altered drug interaction could have a role in AQR to combination therapy, based on the rationale that drug interaction can be a determinant of combination treatment efficacy. To test this hypothesis, models of AQR to either the combination of AZD6244 and BKM120 (HCT116CR), AZD6244 alone (HCT116AR), or BKM120 alone (HCT116BR) were generated through prolonged treatment, along with cells treated with DMSO vehicle (HCT116DM). In support of the hypothesis, CI values revealed the combination of AZD6244 and BKM120 was antagonistic in HCT116CR cells, while it remained synergistic in HCT116DM cells (Table 1). Combination treatment also
A
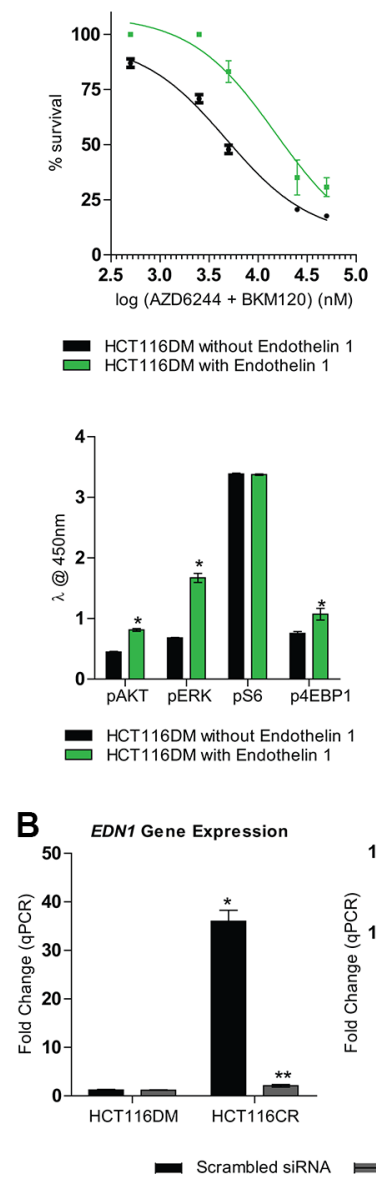

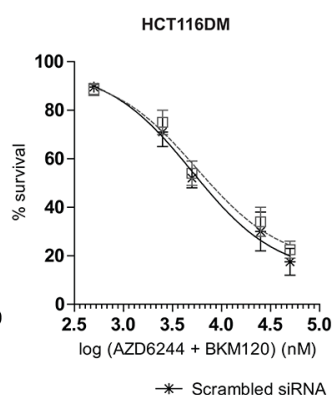

*- Scrambled siRNA
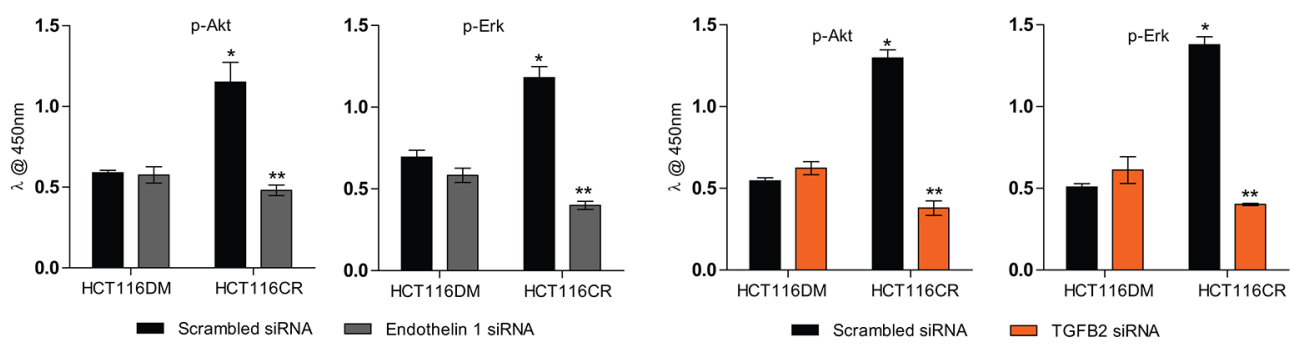

Figure 3: Effect of modulation of EDN1 and TGFB2 in HCT116DM and HCT116CR cells. (A) Concentration response curves (top panel) and pathway signaling (bottom panel) of HCT116DM cells with or without extracellular supplementation of $100 \mathrm{nM}$

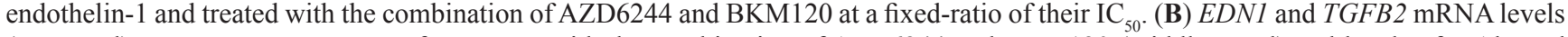
(top panel), Dose response curves of treatment with the combination of AZD6244 and BKM120 (middle panel) and levels of p-Akt and p-Erk (bottom panel) in HCT116DM and HCT116CR cells transfected with scrambled or EDN1 siRNA. (C) EDN1 and TGFB2 mRNA levels (top panel), Dose response curves of treatment with the combination of AZD6244 and BKM120 (middle panel) and levels of p-Akt and p-Erk (bottom panel) in HCT116DM and HCT116CR cells transfected with scrambled or TGFB2 siRNA. All experiments were repeated three times, and data are displayed as mean \pm standard deviation. RNA expression was determined by real-time PCR, and normalized to ACTB levels and ratios in parental HCT116 cells. Protein phosphorylation levels were measured by ELISA, and normalized to total protein levels. *and **indicates $p<0.05$ compared to HCT116DM and HCT116CR controls respectively. 
remained synergistic in HCT116AR and HCT116BR cells, indicating that the loss of synergy was a specific feature of AQR in HCT116CR cells. In addition, pathway signaling analysis revealed that both single agent and combination treatment continued to inhibit expected targets in all cells. This suggested that factors other than loss of direct inhibitory activity were involved in the resistance.

Numerous measures were included in this study to support the validity of the loss of synergy. For single agent treatment, two doses of drugs at $1 / 2 \mathrm{IC}_{50}$ concentrations were used to provide consistency with combination treatment in the number of drug aliquots. Cells with prolonged vehicle treatment were used to control for the effects of treatment over time. Cells were treated with alternative MEK (GDC0973) and PI3K (BYL719) inhibitors and similar trends were observed (Table 1), supporting that the observations were not compound specific. Parallel lines of LoVo cells were generated and similar patterns of resistance were observed in these cells (Supplementary Table S1), indicating that the observations were also not cell line specific. Lastly, tumor xenografts confirmed the resistance phenotype in-vivo (Figure 2).

Many other differences between AQR to combination and single agent treatment were revealed by the study design. Phosphorylation of 4EBP1 was uniquely reduced, and AZD6244 treatment reduced p-Akt and p-4EBP1 in HCT116CR cells but not others (Figure 1). In addition to reduced G1 arrest observed in HCT116AR, HCT116BR and HCT116CR cells, apoptosis was reduced in HCT116CR cells following combination treatment (Figure 2). Cell migration was less impeded by combination treatment in HCT116CR cells compared to all other cells. While HCT116AR cells were more resistant to mTOR inhibitors, HCT116CR cells were more sensitive. These additional phenotypes caution against the assumption that resistance to combination treatment is a composite of resistance to its component agents.
To gain insight into potential mechanisms for the loss of synergy, sequencing of 50 genes frequently mutated in cancer was performed. Unique DNA variants were detected in SMAD4 (Y412H) in HCT116AR, PTEN (A126S) in LoVoAR, and TP53 (T18A) and TP53 (Y236C) in HCT116CR cells. All these variants were predicted to be "deleterious" and "probably damaging" by SIFT [20] and Polyphen [21]. The PTEN (A126S) variant partially inactivates PTEN [22], and thereby could activate PI3K signaling and compensate for MEK pathway inhibition. Inactivation of p53 by TP53 (18A) and TP53 (Y236C) mutation could be involved in $\mathrm{AQR}$, given the role of p53 in tumor suppression and stress response [23]. Nonetheless, these aberrations were not consistent between models, prompting further investigation.

Gene expression array analysis revealed EDN1 and TGFB2 as the top candidate genes overexpressed in both HCT116CR and LoVoCR cells but not in the other cells (Supplementary Figure S2). The EDN1 gene encodes for endothelin-1, a vasoactive peptide, which is typically associated with vasoconstriction and endothelial functions [24]. Endothelin-1 binds to endothelin receptors A and B, which are G-protein coupled receptors (GPCRs), and via second messengers activate many signaling pathways, including MEK and PI3K pathways [24]. Endothelin-1 can also trigger activation of protein kinase $C$ [25], which can lead directly to Erk and Akt activation $[26,27]$. TGF $\beta$ is a well-known cytokine that mediates numerous processes such as proliferation, differentiation, survival, adhesion, and migration through its activation of the SMAD family of proteins [28]. Interestingly, inhibition of MEK and PI3K signaling can lead to TGF $\beta$ activation [29], and EDN1 is a known downstream target of TGF $\beta / \mathrm{SMAD}$ activated transcription $[15,16]$.

Taken together, a mechanism through which HCT116CR and LoVoCR cells AQR to combination treatment can be rationalized (Figure 4). It can be
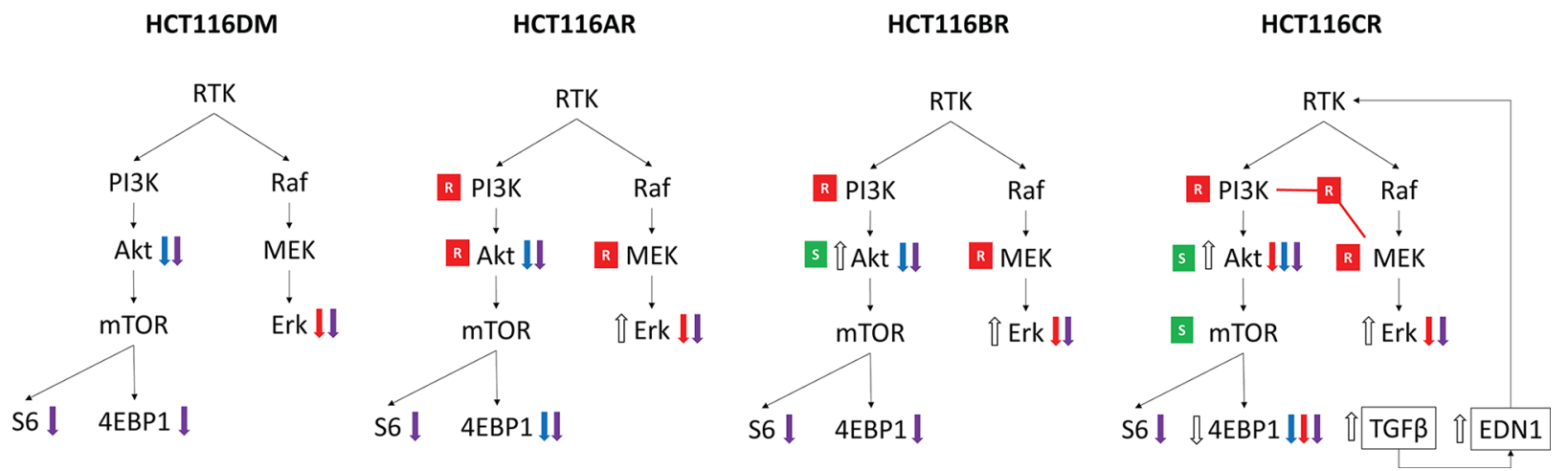

Figure 4: Overview of differences in phenotypes of AQR cells. An increase $(\Uparrow)$ or decrease $(\Downarrow)$ in phosphorylation levels at baseline is indicated by white arrows. An increase $(\Uparrow)$ or decrease $(\downarrow)$ in phosphorylation levels post-treatment are indicated by colored arrows, according to treatment with AZD6244 alone (red), BKM120 alone (blue) and their combination (purple). Resistance (red box with white R) and sensitivity (green box with white S) to inhibitors of respective proteins in drug sensitivity analysis are also indicated. Also indicated is the increased expression of EDN1 and TGFB2 that was observed uniquely in HCT116CR cells, and its hypothesized activation of pathway signaling. 
hypothesized that the combined inhibition of PI3K and MEK pathways led to the activation of TGF $\beta$ and subsequently EDN1. The increased endothelin-1 then provided compensatory activation of the PI3K and MEK pathway, contributing to resistance to combination treatment. In support of this hypothesis, supplementation with endothelin-1 converted the synergism of combination treatment in parental HCT116 and LoVo cells to antagonism, along with activating Akt, Erk and 4EBP1 (Figure 3, Supplementary Figure S5). Silencing of EDN1 or TGFB2 by siRNA also changed the antagonism of combination treatment in HCT116CR and LoVoCR cells to synergism, and reduced p-Akt and p-Erk levels. Additionally, bosentan, a dual endothelin receptor antagonist restored synergy between BKM120 and AZD6244 in HCT116CR and LoVoCR cells (Supplementary Figure S6).

However, it is less clear how a change in drug interaction has occurred. Historically, synergy or antagonism could be understood as an enhancement or reduction of receptor binding or enzyme activity resulting from the combination of two agents [30]. Without a common receptor or enzyme in this study, one approach could be to consider the coordinated inhibition of the MEK and PI3K pathways and its downstream effects as the singular target of drug interaction. With this consideration, the upstream activation of MEK and PI3K pathways by endothelin-1 through GPCRs [31], or activation of Erk and Akt downstream of MEK and PI3K inhibition [5], can be viewed as means to decouple pathway inhibition and/ or bypass inhibitor effects, and thereby alter the singular system. It also remains that endothelin- 1 could activate alternative pathways that modify the downstream events of MEK and PI3K inhibition through its multiplicity of pathways. The reduced apoptosis and inhibition of migration of HCT116CR cells following combination treatment (Figure 2) could be a manifestation of this effect. The unexpected sensitivity of HCT116CR cells to Akt and mTOR inhibitors could be indicative of altered pathway dynamics, while also highlighting a potential rationale for intervention.

In conclusion, the results of this study have supported the hypothesis that an altered drug interaction can determine $\mathrm{AQR}$ to combination treatment. Differences in the phenotypes of AQR to single agent and combination treatment have indicated that caution should be exercised in assuming mechanisms of resistance for combination treatment can be determined from studies of single agent treatment. Overexpression of the TGFB2-EDN1 axis was identified as a potential mechanism of acquired loss of synergy for combination MEK and PI3K inhibitor therapy, and it would be of interest to observe whether this mechanism has a role in clinical resistance as current trials of this combination mature. A precise delineation of the mechanisms of altered drug interaction could not be achieved, as the protocol of Chou and Talalay only informs on the overall nature of interaction and not its components [19]. Systems biology characterization of the complex multiplicity of effectors and pathways in the TGFB2-EDN1 axis may help to shed further light on how drug interactions can affect drug resistance in its modern setting.

\section{MATERIALS AND METHODS}

\section{Generation of cells with acquired resistance}

HCT116 and LoVo colorectal cancer cells were obtained from American Type Culture Collection (Manassas, VA). Cells were cultured in DMEM and supplemented with 10\% fetal bovine serum (Invitrogen, Carlsbad, CA), 50,000 units penicillin and $50 \mathrm{mg}$ streptomycin (Sigma, St Louis, MO) at $37^{\circ} \mathrm{C}$ in a humidified atmosphere containing 5\% $\mathrm{CO}_{2}$. Cell lines were continuously exposed to either a) two aliquots of vehicle (DMSO), b) two aliquots of $1 / 2 \mathrm{IC}_{50}$ concentration of AZD6244, c) two aliquots of $1 \frac{1}{2} \mathrm{IC}_{50}$ concentration of BKM120, or d) aliquots of $\mathrm{IC}_{50}$ concentration of AZD6244 and BKM120 each for a period of 3-8 months. The growth of the cells was monitored weekly and acquisition of resistance was indicated by normal proliferation of cells under the described selection pressures. The cells were maintained with the relevant concentrations of compounds or DMSO respectively. Clonal selection was not carried out for these various AQR cell lines. All the parental and resistant cells passed the authentication testing using short tandem repeat profiling performed by Promega GenePrint ${ }^{\mathbb{B}}$ 10 system (Madison, WI, USA). The cell lines were tested at the commencement and also at the completion of the study.

\section{Drug sensitivity analysis}

AZD6244, BKM120, BYL719, GDC0973, sorafenib, trametinib, BEZ235, Ku-0063794, RAD001, MK2206 and bosentan were obtained from Selleck Chemicals (Houston, TX). Carboplatin and 5-FU were obtained from Sigma. All stock solutions were prepared in DMSO (MP Biomedicals, Solon, $\mathrm{OH}$ ) at a final concentration in culture media of $0.25 \%(\mathrm{v} / \mathrm{v})$. Cells in $90 \mu \mathrm{l}$ medium were seeded (3000 cells/well) onto 96well microtitre plates (Nunc, Rochester, NY). After 24 hours, $10 \mu \mathrm{l}$ of medium containing compounds in graded concentrations ranging from $0.1 \mu \mathrm{M}$ to $1000 \mu \mathrm{M}$ was added to the wells. Control wells contained $20 \mu \mathrm{l}$ of relevant solvent to achieve a final concentration of $0.25 \%$ of each solvent. The effect on cell numbers was assessed using the CellTiter $96^{\circledR}$ AQueous Non-Radioactive Cell Proliferation Assay (Promega, Madison, WI) (MTS Assay) at $72 \mathrm{~h}$ post-treatment. The $\mathrm{IC}_{50}$ was calculated as the drug concentration that inhibited cell proliferation by $50 \%$ compared to vehicle controls as previously described [32]. 


\section{Drug combination analysis}

The effect of combining compounds was evaluated using the median-effect equation and combination index (CI) method of Chou and Talalay [10]. For fixed-ratio experiments the concentrations of each compound to reduce the absorbance to $50 \%$ of that obtained with control cells (inhibitory concentration $50 \% ; \mathrm{IC}_{50}$ ) were generated. Most experiments were performed by combining both agents added together at a fixed 1:1 ratio of the $\mathrm{IC}_{50}$ of each individual drug or a fixed low growth inhibitory dose. The effects of the combination were calculated for each experimental condition using an spreadsheet based on the median-effect analysis method of Chou and Talalay [10]. We have previously described more details of this analysis [33]. For each level of fraction unaffected (fu), a CI was calculated as follows: $\mathrm{CI}=(D)_{1} /\left(D_{\mathrm{f}}\right)_{1}+(D)_{2} /$ $\left(D_{\mathrm{f}}\right)_{2}+\left[(D)_{1}\left(D_{\mathrm{f}}\right)_{2} /\left(D_{\mathrm{f}}\right)_{1}\left(D_{\mathrm{f}}\right)_{2}\right]$, where $(D)_{1}$ and $(D)_{2}$ are the concentrations of the combination required to produce fu, and $\left(D_{\mathrm{f}}\right)_{1}$ and $\left(D_{\mathrm{f}}\right)_{2}$ are the concentrations of the individual drugs required to produce fu. Data giving linear regression coefficients $\left(r^{2}\right)$ of median-effect plots $<0.95$ were excluded. CI values of $<1,1$ and $>1$ were considered to indicate synergy, additivity and antagonism respectively. $\mathrm{CI}$ values with the non-exclusive assumption have been reported.

\section{ELISA analysis}

Levels of p-AKT (Ser473), p-mTOR (Ser2448), p-S6 (Ser235/236), p-4eBP1 (Thr37/46) and their respective total proteins were measured using PathScan ${ }^{\circledR}$ Sandwich ELISA kits (Cell Signaling Technology, Beverly, MA) on the Infinite 200 Pro (Tecan, Mannedorf, Switzerland) according to the manufacturer's instructions.

\section{Cell cycle analysis}

Exponentially growing cells were seeded into $25 \mathrm{~cm}^{2}$ tissue culture flasks at $1 \times 10^{6}$ cells/flask and allowed to attach for $24 \mathrm{~h}$ prior to drug addition. Following $24 \mathrm{~h}$ incubation, both attached and detached cells were collected and fixed with $2 \mathrm{ml}$ of ice-cold $70 \%$ ethanol. Following centrifugation at $900 \mathrm{~g}$ for $5 \mathrm{mins}$, the pellet was resuspended in $800 \mu 1$ of PBS containing $100 \mu \mathrm{l}$ of $1 \mathrm{mg} / \mathrm{mL}$ RNaseA and $100 \mu \mathrm{l}$ of $400 \mu \mathrm{g} /$ $\mathrm{mL}$ propidium iodide (both from Sigma) and stored overnight at $4{ }^{\circ} \mathrm{C}$. Samples were analysed on a LSRII flow cytometer (BD, Franklin Lanes, NJ) equipped with an argon laser tuned to $488 \mathrm{~nm}$ and the red fluorescence collected at $630 \mathrm{~nm}$. The data was analysed using WinMDI v 2.8 and DNA histograms were gated on a display of DNA peak signal against DNA area to exclude debris and clumps.

\section{Apoptosis measurement}

Apoptosis was measured using the Cell Death ELISA $^{\circledR}$ (Roche, Mannheim, Germany) kit. Cells were plated in 96-well plates (3000 cells/well) and on the following day treated with drug or solvent in a volume adjusted to $200 \mu \mathrm{L}$ with $10 \%$ FCS/DMEM. After 24 hours, nucleosomes were quantified according to the manufacturer's instructions.

\section{In vivo assessment}

The study received ethics board approval at the National Cancer Centre of Singapore and Singapore General Hospital. All mice were maintained according to the "Guide for the Care and Use of Laboratory Animals" published by National Institute of Health, USA. They were provided with sterilised food and water ad libitum, and housed in negative pressure isolators with $12 \mathrm{~h}$ light/ dark cycles. HCT116 parental and HCT116CR cells were implanted in both flanks of male SCID mice aged 9-10 weeks. Each injection consisted of approximately $5 \times 10^{6}$ cells. Treatments started when the tumors reached the size of approximately $150-200 \mathrm{~mm}^{3}$. Mice bearing tumors were treated as follows: AZD6244 $(25 \mathrm{mg} / \mathrm{kg})$ + BKM120 (40 mg/kg) p.o. daily, and vehicle controls receiving PEG300/captisol (30:30:water) p.o. daily. Each treatment arm involved 4 independent tumor-bearing mice. The treatment lasted for 10 days. Bi-dimensional measurements were performed twice a week and tumor volumes are calculated based on the following formula: Tumor volume $=\left[(\right.$ Length $) \times\left(\right.$ Width $\left.\left.^{2}\right) \times(\mathrm{p} / 6)\right]$. Tumors in the vehicle-treated groups were harvested on day 10 when the tumor size reached $\sim 1500 \mathrm{~mm}^{3}$. Tumors in AZD6244/ BKM120 group were allowed to grow for an additional 10 days before harvesting. The data were plotted as means and standard errors for each treatment group versus time. At the end of the study, the mice were sacrificed and tumor samples collected.

\section{Mutational analysis}

DNA was extracted from cells using the DNA easy Blood and Tissue kit (Qiagen, Hilden, Germany) and quantified using the Picogeren method (Thermo Fisher, Waltham, MA). Mutation analysis by next generation sequencing was performed using the Ion AmpliSeq Cancer Hotspot Panel v2 (Thermo Fisher) on the Ion Torrent PGM instrument (Thermo Fisher) according to the manufacturer's instruction. This panel consists of 207 amplicons covering approximately $22 \mathrm{~kb}$ of regions in 50 genes with known cancer associations. A total of $2 \mu \mathrm{l}$ DNA were used to generate barcoded libraries using the IonXpress barcode adapters (Thermo Fisher). Amplified libraries were quantified using Tapestation 
High Sensitivity D1000 screentape (Agilent Technologies, Santa Clara, CA) and up to 16 barcoded libraries were combined to a final concentration of $10 \mathrm{pM}$. The pooled libraries underwent amplification by emulsion PCR on Ion Spheres Particles and enrichment using the Ion One Touch System (Thermo Fisher). Sequencing was performed on the Ion Torrent PGM with the 318 chip (Thermo Fisher). Reads were aligned to hg19 and variant called using Ion Torrent Suite 3.6.2 (Thermo Fisher). Variants were annotated using Ensembl Variant Effect Predictor v75 and filtered for variants that were frequent in less than $5 \%$ of the Asian population in the 1000 genomes project, and non-synonymous.

\section{Gene expression analysis}

Total RNA was isolated using the RNeasy Mini kit (Qiagen) and confirmed for quality using the RNA 6000 Nano kit on the Agilent Bioanalyzer 2100 (Agilent Technologies). Gene expression analysis was performed using Affymetrix Human Gene 1.0ST genechips on the Affymetrix Fluidics Station 450 (Affymetrix, Santa Clara, CA). From $200 \mathrm{ng}$ of total RNA, cDNA was generated, fragmented, and biotin labelled using the Applause WTAmp ST system (NuGEN, San Carlos, CA). The prepared targets were hybridized overnight to the arrays, after which the arrays were washed, stained and scanned according to manufacturer's instructions. Affymetrix CEL files were normalized by the Robust Multi-array Average (RMA) method using the R/bioconductor Affy library. We used the TM4-MeV software to perform both the unsupervised hierarchical clustering and differential gene expression via the LIMMA module.

\section{Extracellular endothelin-1 priming}

Exponentially growing HCT116 or LoVo parental cells were seeded in 96 well plates (3000 cells/well). After $24 \mathrm{~h}$, the culture medium was replaced by serum-free medium for an additional $24 \mathrm{~h}$ in the presence or absence of $100 \mathrm{nM}$ endothelin-1 (Sigma). After $24 \mathrm{~h}$ of priming, cells were washed with sterile PBS and re-introduced to normal serum containing medium and combination drug exposure carried out as described in drug sensitivity analysis.

\section{siRNA treatment}

Two independent EDN1 (Cat \# SR301329) and TGFB2 (Cat \#SR304807) siRNA were obtained from Origene Technologies (Rockville, MD). Cells were transfected with siRNA at concentrations of $10 \mathrm{nM}$ and exposed to a range of concentrations of AZD6244, BKM120 or their combination $24 \mathrm{~h}$ posttransfection. The effect on cell numbers was measured at $72 \mathrm{~h}$ post-transfection by the CellTiter $96^{\circledR}$ AQueous Non-Radioactive Cell Proliferation Assay (Promega).
Validation of knockdown efficiency was performed on the ABI7900HT real time system (Thermo Fisher) using Taqman gene expression assay for EDN1, TGFB2 and $A C T B$. Relative quantities were measured by the $\Delta \Delta C \mathrm{~T}$ method [34], using $A C T B$ as a reference gene and parental HCT116 cells as a calibrator.

\section{Statistical analysis}

Differences in $\mathrm{IC}_{50}$ values and protein ELISA were assessed using a paired sample $t$-test. A one-sample $t$-test (two-tailed) was used to compare the $\mathrm{CI}_{\text {fu } 0.5}$ with the predicted value for additivity of 1 . For apoptosis, oneway ANOVA followed by Dunn's Multiple Comparison test was performed to assess for differences. All statistical analyses were two-tailed and performed using GraphPad Prism 5.00 software (GraphPad Software Inc., San Diego, CA). Statistical significance was considered when $p<0.05$.

\section{ACKNOWLEDGMENTS}

This study was supported by the National Research Foundation Singapore and the Singapore Ministry of Education under its Research Centres of Excellence initiative.

\section{CONFLICTS OF INTEREST}

None.

\section{Authors' contributions}

Conception: B. Bhattacharya. Development of Methodology: B. Bhattacharya, R. Soong. Acquisition of data: B. Bhattacharya, SHH Low, ML Chong, D Chia, KK Koh, N Sapari, H Huynh. Analysis and Interpretation of data: (e. g. statistical analysis, bioinformatics): B. Bhattacharya, R. Soong, T. Benoukraf. Writing and Review of Manuscript: B. Bhattacharya, SB Kaye, R. Soong. Study Supervision: B. Bhattacharya, R. Soong.

\section{REFERENCES}

1. Al-Lazikani B, Banerji U, Workman P. Combinatorial drug therapy for cancer in the post-genomic era. Nat Biotechnol. 2012; 30:679-92. doi: 10.1038/nbt.2284.

2. Holohan C, Van Schaeybroeck S, Longley DB, Johnston PG. Cancer drug resistance: an evolving paradigm. Nat Rev Cancer. 2013; 13:714-26. doi: 10.1038/nrc3599.

3. Gottesman MM. Mechanisms of cancer drug resistance. Annu Rev Med. 2002; 53:615-627.

4. Wee S, Jagani Z, Xiang KX, Loo A, Dorsch M, Yao YM, Sellers WR, Lengauer C, Stegmeier F. PI3K pathway 
activation mediates resistance to MEK inhibitors in KRAS mutant cancers. Cancer Res. 2009; 69:4286-4293.

5. Britten CD. PI3K, MEK inhibitor combinations: examining the evidence in selected tumor types. Cancer Chemother Pharmacol. 2013; 71:1395-1409.

6. Roper J, Sinnamon MJ, Coffee EM, Belmont P, Keung L, Georgeon-Richard L, Wang WV, Faber AC, Yun J, Yilmaz OH, Bronson RT, Martin ES, Tsichlis PN, et al. Combination PI3K/MEK inhibition promotes tumor apoptosis and regression in PIK3CA wild-type, KRAS mutant colorectal cancer. Cancer Lett. 2014; 347:204-211.

7. Posch C, Moslehi H, Feeney L, Green GA, Ebaee A, Feichtenschlager V, Chong K, Peng L, Dimon MT, Phillips T, Daud AI, McCalmont TH, LeBoit PE, et al. Combined targeting of MEK and PI3K/mTOR effector pathways is necessary to effectively inhibit NRAS mutant melanoma in vitro and in vivo. Proc Natl Acad Sci U S A. 2013; 110:4015-4020.

8. Haagensen EJ, Kyle S, Beale GS, Maxwell RJ, Newell DR. The synergistic interaction of MEK and PI3K inhibitors is modulated by mTOR inhibition. Br J Cancer. 2012; 106:1386-1394.

9. Shimizu T, Tolcher AW, Papadopoulos KP, Beeram M, Rasco DW, Smith LS, Gunn S, Smetzer L, Mays TA, Kaiser B, Wick MJ, Alvarez C, Cavazos A, et al. The clinical effect of the dual-targeting strategy involving PI3K/ $\mathrm{AKT} / \mathrm{mTOR}$ and RAS/MEK/ERK pathways in patients with advanced cancer. Clin Cancer Res. 2012; 18:2316-2325.

10. Chou TC, Talalay P. Quantitative analysis of dose-effect relationships: the combined effects of multiple drugs or enzyme inhibitors. Adv Enzyme Regul. 1984; 22:27-55.

11. Little AS, Balmanno K, Sale MJ, Newman S, Dry JR, Hampson M, Edwards PA, Smith PD, Cook SJ. Amplification of the driving oncogene, KRAS or BRAF, underpins acquired resistance to MEK1/2 inhibitors in colorectal cancer cells. Sci Signal. 2011; 4:ra17.

12. Yuen JS, Sim MY, Sim HG, Chong TW, Lau WK, Cheng CW, Ong RW, Huynh H. Combination of the ERK inhibitor AZD6244 and low-dose sorafenib in a xenograft model of human renal cell carcinoma. Int J Oncol. 2012; 41:712-720.

13. Chang L, Graham PH, Hao J, Ni J, Bucci J, Cozzi PJ, Kearsley JH, Li Y. PI3K/Akt/mTOR pathway inhibitors enhance radiosensitivity in radioresistant prostate cancer cells through inducing apoptosis, reducing autophagy, suppressing NHEJ and HR repair pathways. Cell Death Dis. 2014; 5:e1437. doi: 10.1038/cddis.2014.415.

14. Forbes SA, Beare D, Gunasekaran P, Leung K, Bindal N, Boutselakis H, Ding M, Bamford S, Cole C, Ward S, Kok CY, Jia M, De T, et al. COSMIC: exploring the world's knowledge of somatic mutations in human cancer. Nucleic Acids Research. 2015; 43:D805-D811.

15. Bellam N, Pasche B. Tgf-beta signaling alterations and colon cancer. Cancer Treat Res. 2010; 155:85-103.
16. Rodriguez-Pascual F, Reimunde FM, Redondo-Horcajo M, Lamas S. Transforming growth factor-beta induces endothelin-1 expression through activation of the Smad signaling pathway. J Cardiovasc Pharmacol. 2004; 44:S3942.

17. Ahronian LG, Sennott EM, Van Allen EM, Wagle N, Kwak EL, Faris JE, Godfrey JT, Nishimura K, Lynch KD, Mermel CH, Lockerman EL, Kalsy A, Gurski JM, et al. Clinical Acquired Resistance to RAF Inhibitor Combinations in BRAF-Mutant Colorectal Cancer through MAPK Pathway Alterations. Cancer Discov. 2015; 5:358-367.

18. Pirazzoli V, Nebhan C, Song X, Wurtz A, Walther Z, Cai G, Zhao Z, Jia P, de Stanchina E, Shapiro EM, Gale M, Yin R, Horn L, et al. Acquired resistance of EGFR-mutant lung adenocarcinomas to afatinib plus cetuximab is associated with activation of mTORC1. Cell Rep. 2014; 7:999-1008.

19. Chou TC. Drug combination studies and their synergy quantification using the Chou-Talalay method. Cancer Res. 2010; 70:440-446.

20. Ng PC, Henikoff S. SIFT: Predicting amino acid changes that affect protein function. Nucleic Acids Res. 2003; 31:3812-3814.

21. Adzhubei IA, Schmidt S, Peshkin L, Ramensky VE, Gerasimova A, Bork P, Kondrashov AS, Sunyaev SR. A method and server for predicting damaging missense mutations. Nat Methods. 2010; 7:248-249.

22. Rodriguez-Escudero I, Oliver MD, Andres-Pons A, Molina M, Cid VJ, Pulido R. A comprehensive functional analysis of PTEN mutations: implications in tumor- and autism-related syndromes. Human molecular genetics. 2011; 20:4132-4142.

23. Zilfou JT, Lowe SW. Tumor suppressive functions of $\mathrm{p} 53$. Cold Spring Harb Perspect Biol. 2009; 1:a001883.

24. Rosano L, Spinella F, Bagnato A. Endothelin 1 in cancer: biological implications and therapeutic opportunities. Nature reviews Cancer. 2013; 13:637-651.

25. Clerk A, Bogoyevitch MA, Anderson MB, Sugden PH. Differential activation of protein kinase $\mathrm{C}$ isoforms by endothelin-1 and phenylephrine and subsequent stimulation of p42 and p44 mitogen-activated protein kinases in ventricular myocytes cultured from neonatal rat hearts. J Biol Chem. 1994; 269:32848-32857.

26. Ueda Y, Hirai S, Osada S, Suzuki A, Mizuno K, Ohno S. Protein kinase $\mathrm{C}$ activates the MEK-ERK pathway in a manner independent of Ras and dependent on Raf. J Biol Chem. 1996; 271:23512-23519.

27. Li W, Zhang J, Flechner L, Hyun T, Yam A, Franke TF, Pierce JH. Protein kinase C-alpha overexpression stimulates Akt activity and suppresses apoptosis induced by interleukin 3 withdrawal. Oncogene. 1999; 18:6564-6572.

28. Stow LR, Jacobs ME, Wingo CS, Cain BD. Endothelin-1 gene regulation. FASEB J. 2011; 25:16-28. 
29. Pardali K, Moustakas A. Actions of TGF-beta as tumor suppressor and pro-metastatic factor in human cancer. Biochimica et biophysica acta. 2007; 1775:21-62.

30. Barrington WW, Jacobson KA, Stiles GL. Demonstration of distinct agonist and antagonist conformations of the A1 adenosine receptor. J Biol Chem. 1989; 264:13157-13164.

31. Bhalla A, Haque S, Taylor I, Winslet M, Loizidou M. Endothelin receptor antagonism and cancer. Eur J Clin Invest. 2009; 39:74-77.

32. Bhattacharya B, Low SH, Soh C, Kamal Mustapa N, Beloueche-Babari M, Koh KX, Loh J, Soong R. Increased drug resistance is associated with reduced glucose levels and an enhanced glycolysis phenotype. Br J Pharmacol. 2014; 171:3255-3267.
33. Bhattacharya B, Akram M, Balasubramanian I, Tam KK, Koh KX, Yee MQ, Soong R. Pharmacologic synergy between dual phosphoinositide-3-kinase and mammalian target of rapamycin inhibition and 5-fluorouracil in PIK3CA mutant gastric cancer cells. Cancer Biol Ther. 2012; 13:34-42.

34. Livak KJ, Schmittgen TD. Analysis of relative gene expression data using real-time quantitative PCR and the 2(-Delta Delta C(T)) Method. Methods. 2001; 25:402-408. 